HIV/STIs campaigns need to improve the sex workers awareness and treatment seeking behaviour for STIs.

\section{P3.417 ASSOCIATION OF SITUATIONAL DRINKING WITH UNPROTECTED ANAL INTERCOURSE (UAI) AMONG MEN WHO HAVE SEX WITH MEN (MSM) AND TRANSGENDER WOMEN (TW) IN URBAN LIMA, PERU (2008)}

doi:10.1136/sextrans-2013-051184.0868

${ }^{1}$ R G Deiss, ${ }^{2}$ R R Segura, ${ }^{2} \mathrm{~J} \mathrm{~L} \mathrm{Clark,}{ }^{2} \mathrm{~K}$ A Konda, ${ }^{3} \mathrm{~S}$ R Leon, ${ }^{3} \mathrm{C}$ A Caceres, ${ }^{2} \mathrm{~T} \mathrm{~J}$ Coates. 'Division of Infectious Diseases, Harbor-UCLA Medical Center, Torrance, CA, United States; ${ }^{2}$ Center for World Health, Department of Medicine, Division of Infectious Diseases, University of California, Los Angeles., Los Angeles, CA, United States; ${ }^{3}$ Unit of Health, Sexuality and Human Development, Cayetano Heredia University School of Public Health, Lima, Peru

Background The association between general patterns of alcohol intake and sexual risk behaviours among men who have sex with men (MSM) and transgendered women (TW) populations has been well- established. However, there is limited event-level data on alcohol use and contact-specific risk behaviour from low resource settings with MSM/TW-concentrated HIV epidemics such as Peru.

Methods We performed secondary analysis of baseline data from a community-based HIV prevention trial with MSM/TW subjects from 24 neighbourhoods (clusters) in urban Lima. Using multivariate Poisson regression with standard errors estimated for clustered data to calculate prevalence ratios (PR), we measured the adjusted association between situational drinking and unprotected anal intercourse (UAI) during the subject's last sexual contact.

Results A total of 718 subjects were included (Mean age $=29$, Range $=18-45 ; 71.1 \%$ MSM and $28.9 \%$ TW), of whom $63.3 \%$ had engaged in transactional sex during the previous year. Overall, $45.4 \%$ of subjects reported UAI with their most recent partner, and $46.1 \%$ reported alcohol intake before their last sexual encounter (drinking either with or without him/her). In multivariate analysis, participants were more likely to practise UAI in the last sexual encounter with their last partners if they drank alcohol before sex ( $\mathrm{PR}=1.23,95 \% \mathrm{CI}: 1.03-1.47)$, after adjusting for age, education, sexual identity, sexual role, number of partners over the last year, transactional sex during the previous year and partner type (stable, casual, or transactional sex partner).

Conclusions The independent association between contact-specific alcohol consumption before sex and UAI among MSM/TW in Peru warrants further in-depth exploration to understand the exact mechanisms and determinants of these two behaviours at individual-, partner- and community-levels. Interventions to reduce sexual risk behaviours should address situational drinking among MSM and TW populations

\section{P3.418 FACTORS ASSOCIATED WITH CONDOM BREAKAGE AMONG MEN WHO HAVE SEX WITH MEN IN INDIA}

doi:10.1136/sextrans-2013-051184.0869

S Ramesh, P Mehrotra, D Ganju. Population Council, New Delhi, India

Background Condom use has long been identified as one of the most effective means of preventing HIV/STI. However recent studies among female sex workers suggest high rates of condom breakage. There is paucity of evidence on the extent of condom breakage, its association with the prevalence of HIV/STI and the factors associated with condom breakage among men who have sex with men (MSM). Methods Data were drawn from a cross-sectional bio-behavioural survey of 3806 self-identified MSM from three high prevalence states of India, recruited through probability based sampling in 2009-10. Logistic regression models were used to examine the association between condom breakage and the prevalence of HIV/STI and to identify the correlates of condom breakage.
Results Overall, $20 \%$ of MSM reported condom breakage in the month prior to the survey. Condom breakage was associated with increased odds of HIV/STI. Additionally, no significant difference in the prevalence of HIV/STI was observed between MSM who were inconsistent condom users and MSM who used condoms at every sexual encounter but reported condom breakage.MSM who were 25-34 years old; were predominantly receptive sexual partner; travelled outside their current place of residence and had sex at destination; consumed alcohol in the month prior to the survey; reported drug use (self or partner); had same sex sexual debut at a younger age and used oil based lubricant were significantly more likely to report condom breakage compared to their counterparts.

Conclusion Condom breakage was common among MSM in this study. Interventions with MSM in India which focus primarily on consistent condom use should also consider the threat posed by condom breakage. Special effort is required to provide information on correct condom use to MSM at higher risk of experiencing condom breakage.

\section{P3.419 EFFECT OF INTERVENTION EXPOSURE ON SELF-REPORTED CONDOM USE AMONG MEN WHO HAVE SEX WITH MEN IN SOUTHERN INDIA}

doi:10.1136/sextrans-2013-051184.0870

'K M Mitchell, 'A M Foss, 2,3B M Ramesh, ${ }^{2} \mathrm{R}$ Washington, ${ }^{2} \mathrm{~S}$ Isac, ${ }^{3} \mathrm{~J} \mathrm{~F}$ Blanchard, ${ }^{3} \mathrm{~S}$ Moses, ${ }^{5} \mathrm{M}$ Boily, ${ }^{6} \mathrm{M}$ Alary, 'P Vickerman. 'London School of Hygiene and Tropical Medicine, London, UK; ${ }^{2}$ Karnataka Health Promotion Trust, Bangalore, India; ${ }^{3}$ University of Manitoba, Winnipeg, MB, Canada; ${ }^{4}$ St. John's Research Institute, Bangalore, India; ${ }^{5}$ Imperial College London, London, UK; ${ }^{6}$ Centre de recherche du CHU de Québec, Québec, OC, Canada

Background The Avahan HIV prevention intervention programme promotes condom use amongst men who have sex with men (MSM) in high HIV prevalence states in India. We assessed how self-reported condom use varies with intervention exposure for MSM in Bangalore.

Methods Self-reported condom use and intervention exposure data came from a 2006 cross-sectional survey of MSM Consistent condom use (CCU) with all, main, and casual male sex partners was assessed. Intervention exposure was measured by: whether MSM had been contacted by Avahan; duration since first contact; number of contacts in the past month; number of condoms received (log-transformed, as plots suggested a non-linear relationship); and number of condom demonstrations seen in the past month. Logistic regression was used to assess the relationship between each exposure variable and CCU with each partner type independently, controlling for demographic, socio-economic and behavioural factors associated with condom use.

Results $77 \%$ of MSM contacted by Avahan reported CCU with all partners, compared with $55 \%$ of those not contacted ( $p=0.019$, adjusted analysis). CCU was not associated with duration since first intervention contact or with number of contacts by the intervention in the past month. CCU with all partners and casual (but not main) partners increased with the number of condoms received. CCU increased with the number of condom demonstrations seen in the last month, for all, main and casual partners (odds of CCU with each partner type increased by 1.7-2.4-fold for each additional condom demonstration, $\mathrm{p}<0.001$ overall).

Conclusion Direct contact with the Avahan programme is associated with increased reported condom use amongst MSM in Bangalore. Although there is potential for social desirability bias, higher levels of reported condom use are associated with repeated contacts with the programme, particularly those involving demonstrations of correct condom use, and with receiving large numbers of condoms from the programme. 